\title{
20 Moderne Forschungsverfahren und ihr Widerstreit im Zeichen der Würde: Helmuth Plessners erste Transformation der kantischen Kritik der Urteilskraft (1920)
}

\subsection{Einleitung}

Helmuth Plessners Auseinandersetzung mit der Philosophie Immanuel Kants durchzieht sein gesamtes Lebenswerk, von seiner ersten philosophischen Publikation Die wissenschaftliche Idee. Ein Entwurf über ihre Form (Plessner 1980a), die 1913 erschien, als er 21 Jahre alt war, über seine Dissertation von 1916 Krisis der transzendentalen Wahrheit am Anfang (Plessner 1980d) bis zu seinem Aufsatz Kants Kunstsystem der enzyklopädischen Propädeutik (1976), als er 84 Jahre alt war, und wo es noch immer heißt: „Kant verstehen heißt über ihn hinausgehen“ (Plessner 1981c, 439). Es ist hier nicht möglich, ein gesondertes Buch über die lohnenswerte Thematik der Transformation der Kantischen Philosophie in Plessners Philosophischer Anthropologie insgesamt vorzulegen (zu dieser Aufgabe Krüger 2001, Krüger 2017d). Plessner nimmt durchgängig Kants Art und Weise auf, in Grenzbegriffen und in Funktionen zu denken. Ich begnüge mich im Folgenden damit, diese Transformation an Plessners Habilitationsschrift Untersuchungen zu einer Kritik der philosophischen Urteilskraft aus dem Jahre 1920 (Plessner 1981e) zu erörtern, weil sie der Urteilskraft, deren Problematik die ganze Kantsche Philosophie durchzieht, gewidmet ist und weil Plessners Transformation genau an ihr, der Urteilskraft, ansetzt: „Wie Plessner bei Kant im Urteil den Kreis entdeckte, aber gleichzeitig die Basis und die Grenze seiner Philosophie, so nimmt er auch selbst einen solchen Kreis a priori in sein System auf" (Redeker 1993, 82).

Plessner hat seine eigene Konzeption von qualitativer Erfahrung, die er phänomenologisch Anschauung nannte, erst von seinem Buch Die Einheit der Sinne. Eine Ästhesiologie des Geistes (1923, hier Plessner 1980b) bis zu seinem Buch Die Stufen des Organischen und der Mensch (1929, hier Plessner 1975) entwickelt und durchgeführt. Dadurch wird die Ermöglichung der Ausbildung von Urteilen in die Lebensführung von Personen vorverlegt, wodurch auch die Begründung von Urteilen in die Kontexte des personalen Lebens eingebettet wird, was ich die performative Wende seiner Philosophischen Anthropologie genannt habe (Krüger 2001, 248). Lebenserfahrung, so Plessner, sei eine qualitative Einheit vom Außen und Innen schon ihres Gegenstandes, erst recht auf Seiten derjenigen, die diese Erfahrung machen. Sie sei nicht mit derjenigen nötigen 
Restriktion zu verwechseln, die in der naturwissenschaftlichen Laborerfahrung dadurch erfolgt, dass sie auf ein vom Innen abgetrenntes Außen des Gegenstands fokussiert wird, weil man dies allein messen kann (Plessner 1975, 26-30, 118-120). Plessner konvergiere, so schon Redeker, alle üblichen Divergenzen zwischen Außen-Innen, Subjekt-Objekt, Form-Materie, Denken-Wahrnehmen, Psyche-Physis. Dadurch treten an die Stelle der dualistischen Fixierungen von Gegensätzen lebendige Spektren von verschieden proportionierten Grenzübergängen. Plessner gelinge ein großer „Kunstgriff“, den er den „Doppelaspekt der Anschauung“ nannte und durch den Plessner den „Konvergenzpunkt von Kant (Urteil), Dilthey (Dichotomie) und Husserl (Phänomen)“ entdecken konnte (Redeker 1993, 88). Man muss diesen Konvergenzpunkt vor Augen haben, um $\mathrm{zu}$ wissen, wohin Plessners philosophischer Weg führen wird. Aber damit er ihn überhaupt antreten konnte, musste er Kants Weg als offene Aufgabe rekonstruiert haben. Plessner stellte auch noch in seinem naturphilosophischen Hauptwerk Die Stufen des Organischen und der Mensch (1928) den Weg seiner „Neuschöpfung der Philosophie“ ausdrücklich in Analogie zu Kants Weg dar (Plessner 1975, 30-31).

Bei allem Neuen, das Plessners Transformation der Kantschen Urteilskraft in die personale Lebensform erbringen wird, sie bleibt doch der Kantschen Spezifik des Philosophieverständnisses verpflichtet. Philosophie habe die Würde des Vernunftwesens Mensch auch und gerade dann zu bewahren, wenn dieses Vernunftwesen mit dem Naturwesen Mensch in der Lebensform von Personen integriert wird. Plessners Transformation der Kantschen Philosophie hält auch später an demjenigen Philosophieverständnis fest, mit dem diese Transformation in Plessners Habilitationsschrift begonnen hat: Im Philosophieren werde die Idee der Würde von Personen bewahrt. Auch daher ist es berechtigt, die größere Aufgabe, Plessners Transformation von Kants Philosophie zu erforschen, mit der kleineren Aufgabe zu beginnen, welchem Philosophieverständnis Plessner auch später treu bleiben wird, so sehr es weiteren Modifikationen unterliegen wird. Diese Frage ist wichtig, weil man ansonsten nicht versteht, worin das Philosophische in seiner Philosophischen Anthropologie besteht.

Helmuth Plessner sieht in seiner Habilitationsschrift Untersuchungen zu einer Kritik der philosophischen Urteilskraft (1920) die Philosophie grundsätzlich in einer weltgeschichtlich neuen Lage zu einer erneuten Selbstbestimmung herausgefordert. Der Neukantianismus gehe aus Überreife seiner Auflösung entgegen. Sein Führungsanspruch werde ihm durch Edmund Husserls Phänomenologie und Wilhelm Diltheys geschichtliche Lebensphilosophie streitig gemacht. Den exakten Naturwissenschaften seien Geschichte und Kulturwissenschaft im Wissenschaftsgefüge zur Seite getreten, so dass die Fundamente 
des Wissenschaftsbegriffes selbst in Revision gingen (siehe Plessner 1981e, 21-24). Die Geschichte der Philosophie erscheine nicht mehr, woran sich Kant insbesondere orientiert hatte, als Antwort auf die Entwicklung der Physik Galileis und Newtons durch die philosophische Grundlegung einer durch Vernunft voranschreitenden Wissenschaft, sondern als „die Geschichte der Auseinandersetzung der fundamentalen Kontraste in Weltanschauung und Lebenswillen durch die Machtmittel der vernünftigen Beweisführung“ (ebd., 17). Inzwischen gehe es auch nicht mehr nur um die bekannten Schwierigkeiten der Kantschen Philosophie, ihre Vermögenspsychologie im Hinblick auf den „Phänomenalismus der transzendentalen Ästhetik und das Ding-an-sich-Problem, die Kategorientafel, den Schematismus und die Dialektik“ (ebd., 21), sondern um das „Prinzip des Systems“, mithin um die „Idee einer wissenschaftlichen Philosophie selbst“ (ebd., 26). Da inzwischen Philosophien als Weltanschauungen in phänomenologischen Typen (Dilthey) oder in Karl Jaspers' Psychologie der Weltanschauungen aufzugehen scheinen, müsse man endlich eine philosophische „Kritik der Philosophie“ selbst unternehmen (ebd., 27). „Diese innere Überwindung Kants wird den Weg freimachen nicht zuletzt $\mathrm{zu}$ einem neuen Wissenschaftsbegriff, der die Geisteswissenschaften in gleicher Weise tragen kann wie die in großartiger Umwälzung begriffene mathematische Naturwissenschaft und Biologie“ (ebd., 30). - Plessner war nicht nur Sohn eines Arztes, sondern auch selbst Biologe, der sowohl empirisch als auch theoretisch mit anderen Biologen zusammengearbeitet hatte, während und bevor er auch zu einem Philosophen geworden war.

Plessner vertrat, um das Ergebnis seiner Habilitation vorwegzunehmen, die Idee einer Philosophie in systematischer Form, indem er das Verhältnis zwischen bestimmender und reflektierender Urteilskraft bei Kant für die Zukunftsorientierung moderner Forschung umkehrt. Die moderne Forschung als Innovation in der Zukunft historisiert dasjenige, das bisher als universell gegolten hat, und versucht dasjenige, das bisher als subjektiver und hypothetischer Geltungsanspruch gegolten hat, $\mathrm{zu}$ universalisieren. Plessner funktionalisiert Kants reflektierende Urteilskraft für moderne Forschung $\mathrm{zu}$ einem Verfahren um. Aber diese Funktionalisierung könne je nach einer verschiedenen Proportion in der reflektierenden Urteilskraft erfolgen. Sie führe daher in einen Widerstreit zwischen den verschieden bestimmbaren Forschungsverfahren. Inmitten dieses Widerstreits, der schon bei Kant mehr und anderes als ein Widerspruch war, setze die erneute Spezifikation des Philosophierens ein. Im zweiten Abschnitt des vorliegenden Beitrages rekonstruiere ich Plessners Vorschlag, die Wissenschaften im modernen Sinne als ein Forschungsverfahren zu begreifen, das nicht nur die Naturwissenschaften, sondern auch die Kultur- und Biowis- 
senschaften und in gewisser Weise überhaupt den Forschungscharakter moderner soziokultureller Unternehmungen ermöglicht. Im dritten Teil meines Beitrags gehe ich Plessners Verständnis von der Spezifik des Philosophierens aus dem Widerstreit zwischen den funktional verschieden proportionierten Forschungsverfahren nach. Er hält die philosophische Begrenzung des Widerstreits durch die Wahrung der Würde von Personen für unabdingbar, wenngleich sich das Philosophieren geschichtlich in der Differenz zwischen dieser Idee des Philosophierens und dem Ideal des jeweiligen philosophischen Systems entfalte. Der Widerstreit wird durch Kritik, d.h. die Aufdeckung dieser Differenz in Gang gehalten. Schließlich deute ich im 4. Abschnitt meines Beitrages an, wie Plessner an der Orientierung auf die Bewahrung der Würde von Personen auch in seinen späteren Werken durch Modifikationen seines Ideals festhält.

\subsection{Die Funktionalisierung der reflektierenden Urteilskraft für moderne Verfahren der Erforschung}

In seinem Wissenschaftsverständnis trägt Plessner dem Forschungscharakter moderner Wissenschaft ${ }^{1}$ Rechnung, den er durch den „Arbeitsbegriff“ im Gegensatz zum „Offenbarungsbegriff der Wissenschaft“ hervorhebt. Wissenschaftlicher Sinn werde in Kontexten der „Entdeckung und Erfindung“ nach methodischen Regeln der Beobachtung und des Experimentes „gemacht“ (Plessner 1981e, 13, 19, 25, vgl. auch 75f., 160). Dafür brauche man ein Verfahren, so argumentiert Plessner durchgängig, das durch Funktionsteilung und deren Reintegration die Auflösung des folgenden Zirkels ermögliche: Man könne diejenigen Maßstäbe, nach denen man schon immer in der Forschung urteilen müsste, erst in der Forschung selber erzeugen. Das Verfahren müsse urteilsfähig machen, daher Plessners Konzentration auf den Funktionskreis des Urteilens in Kants Vermittlung seiner Philosophie unter dem Primat des Praktischen. „Erst das Urteil verknüpft in einem eigenen Grenzübergang eine bestimmte Sache (als Subjekt) mit einer anderen bestimmten Sache (als Prädikat) synthetisch und geht über die Grenze hinüber, die der Begriff im Bezeichnen erst zieht“ (ebd., 106).

Plessner denkt hier semiotisch, was man im Sinne des „crossing boundary“ von George Spencer-Brown auch aktuell lesen kann. Semiotisch betrachtet ver-

1 Vergleiche zum Doppelaspekt der Forschungs- und Darstellungsrationalität in der europäischen Rationalitätstradition Mittelstraß 1989, 260-266. 
steht Plessner die Ermöglichung des Urteilens als einen Grenzübergang (crossing boundary). Nachdem begrifflich eine Unterscheidung durch eine Darstellungstechnik bezeichnet (nicht nur dem Worte nach benannt) worden ist, wird diejenige Seite der Unterscheidung markiert, auf der man nunmehr operiert. Von der markierten Seite her wird die Unterscheidung verwendet und angesichts der in der Prädikation enthaltenen Verwendung zur Aufgabe der Beurteilung. Die Erfüllung dieser Aufgabe erfordert einen methodischen Übergang über die gezogenen Grenzen hinaus in die begrifflich markierten Grenzen (siehe Spencer-Brown 1979, 2-5), deren Grenzübergänge grammatikalisch nach ihrer Rolle als Subjekt oder als Prädikat von Sätzen verknüpft werden.

Da es sich zudem in der Forschung nicht um die Abarbeitung bekannter Gesetzmäßigkeiten oder Regeln handeln kann, sondern um die Entdeckung und Erfindung von Neuem dreht, fokussiert Plessner auf die reflektierende Urteilskraft. Im Unterschied zur bestimmenden Urteilskraft, die Besonderes unter gegebenes Allgemeines subsumiert, habe die reflektierende Urteilskraft die folgende heuristische Funktion zu erfüllen: Ausgehend von einer gegebenen Zufälligkeit, Besonderheit und Subjektivität werde das dazu passende allgemein und objektiv Gültige erst gesucht, indem in Analogie zu einem bestimmten Zweck Variationen durchgespielt werden, als ob es diesen Zweck allgemein und objektiv gäbe (im Konjunktiv). Die reflektierende Urteilskraft kann daher für die Forschungsgegenstände keine konstitutive, wohl aber eine in dem Forschungsverfahren regulative Bedeutung entfalten (siehe Kant 1908, 179-184).

Im Falle der ästhetisch reflektierenden Urteilskraft führen Gefühle der Lust oder Unlust, die anlässlich bestimmter Phänomene hervorgerufen werden, zu Geschmacksurteilen, die subjektiv verallgemeinerbaren Anspruch auf Zustimmung im Gemeinsinn erheben. Obgleich Geschmacksurteile nicht zu beweisen sind, kann für sie aber doch immerhin gestritten werden und können sie bestritten werden (siehe Kant 1908, § 20 u. § 56). Sie betreffen die subjektive Verallgemeinerung der Möglichkeit eines Zusammenspiels von Funktionen, das für eine objektiv allgemeingültige Erkenntnis nötig wäre. Insofern machen solche ästhetischen Geschmacksurteile als Ausgangspunkt Hoffnung darauf, durch Reflexion auf ihre Ermöglichung der Forschungsaufgabe, Phänomene auf bestimmte Weise anschaulich darstellbar werden zu lassen, vor- und zuzuarbeiten. Bezieht man die ästhetisch reflektierende Urteilskraft auf die Produktion von Neuem, dann betrifft sie funktional gesehen die Aufgabe der anschaulichen Darstellung.

Im Unterschied zu der ästhetisch reflektierenden Urteilskraft setzt die teleologisch reflektierende Urteilskraft nicht bei der anschaulichen Darstellungsaufgabe, sondern bei der Erklärungsaufgabe der Forschung an, die bis zu einer Technik der Erzeugung führen kann (Kant 1908, § 78). Insbesondere im Hinblick 
auf sich selbst organisierende Wesen, wie schon Kant das Anschauungsphänomen von Lebewesen in seiner Kritik der Urteilskraft beschreibt, reiche die mechanische Erklärungsweise der Naturkausalität nicht aus, dieser Phänomenart in einer angemessenen Proportion der Erkenntnisfunktionen gerecht werden zu können. Einerseits komme es in einem sich selber organisierenden Lebewesen zu einer Wechselseitigkeit von Ursache und Wirkung, die in den Zirkel von Selbstverursachung und Selbstbewirkung führe. Andererseits gehe aber die Ganzheitlichkeit von Organismen nicht aus der Summierung ihrer Teile hervor, wie sich auch umgekehrt Organe nicht wie Teile des Organismus behandeln lassen, ohne dass der Organismus als ganzer Schaden nähme. Angesichts dieser Probleme rufen wir unser teleologisches Selbstverständnis als zweckmäßig und selbstzweckhaft handelnde Wesen auf, die den Bedingungen angemessene Mittel für die gesetzten Zwecke beziehungsweise Selbstzwecke verwenden können. Gleichwohl wissen wir, dass wir unsere eigene Teleologie weder der Natur als ganzer noch bestimmten ihrer Naturprodukte als bestimmte Naturzwecke andichten dürfen (siehe Kant 1908, § 65). Die Reflexion auf die Übertragung der Teleologie kann nur der Hypothesengewinnung dienen, der Gewinnung von Hypothesen derart, $a l s o b$ sich selber organisierende Wesen eine übersummenhafte Ganzheit wären und sich als Selbstzweck setzen könnten (im Konjunktiv).

Der funktionsteilige Charakter des Forschungsverfahrens kommt bei Plessner in der Unterscheidung grammatikalischer und logischer Verwendungsweisen von Sprache in der Wissenschaft zum Ausdruck. Im Anschluss an Wilhelm von Humboldt hebt er „das pragmatische Problem vom Ursprung der Sprache, d. i. die Möglichkeitsbedingungen des Sprachphänomens, also das kritische Problem der Sprache“ (Plessner 1981e, 107) hervor, das er grammatikalisch als den Grenzübergang zwischen Subjekt und Prädikat fasst. Während die theoretischen Urteile relational in ihrer logischen Form „zum hypothetischen Kreis“ gehörten, müssten sie grammatikalisch, sprich pragmatisch gesehen in dem Forschungsverfahren erst die „Modalitäten ihrer Gewissheit“, ob sie nämlich problematisch, assertorisch oder apodiktisch genommen werden dürfen, durchlaufen: „In der Form der Beziehung zwischen Subjekt und Prädikat gelten andere Gesetze als in der Weise der Gewissheit, mit der die Aussage fällt“ (ebd., 16). Pragmatisch gesehen müsse das Verfahren ermöglichen, dass die „Bedingungen des Ansinnens“, das man an andere stellen kann, „zugleich die Bedingungen der Einstimmung oder wenigstens der entschiedenen Stellungnahme dazu“ (ebd., 33) sind. Der traditionell grammatikphilosophische Zugriff wird von Plessner in eine sprachpragmatische Fassung der Rollenverteilung überführt, in der man für die Schaffung der Bedingungen des Ansinnens unter den Bedingungen der Einstimmung Verantwortung übernehmen kann. In der Gegen- 
wartsphilosophie hat Brandom für die Lösung dieser Aufgabe einen entsprechend normativen Pragmatismus entwickelt (Brandom 1994).

Was den Formalismus der Forschung, der die nötige Vertretbarkeit von Elementen in der Prozedur sichere, näher angeht, unterscheidet Plessner zwischen dem diskursiv operativen Formalismus und dem für die Anschauung intuitiven Formalismus. „Beide Male liegt dasselbe Grenzverhältnis zu Grunde, nur wird es als gleichwertig nach einem Verfahren [dem diskursiv-operativen Verfahren: HPK], als gleichartig in der Anschauung beurteilt“ (Plessner 1981e, 120). Das diskursiv operative Verfahren führe entfaltet zum „Rechnen“ und die Formung der Anschauung zur „Kontur“ der „Zeichnung“. Für den Übergang zwischen dem diskursiv-operativen Formalen und dem für die Anschauung intuitiven Formalen werde „die seelische oder auch körperliche Natur unseres Daseins ins Spiel gesetzt“: Wozu jedoch „die Bedingungen alles Spiels selbst zureichen, das ist subjektiv allgemein gültig, aber nur von Subjekt zu Subjekt, niemals intersubjektiv“ (ebd., 123). Daher müsse das wechselseitige Genügen der „Bedingungen der Zeichnung und der Rechnung“ füreinander nach dem „Typus der Analogie“ zu einer intersubjektiv allgemeinen „Übereinstimmung zwischen Objekt und logischer Form“ gebracht werden (ebd., 125). Dies geschehe methodisch durch eine figürliche Schematisierung der Anschauung zur Wahrnehmung und durch eine analogisierende Typisierung des Diskurses (ebd., 131). Man probiere die wechselseitige Passung von Wahrnehmung und Diskurs solange aus, bis das anfangs zufällige und subjektive Zusammenspiel zu einem methodisch vertretbaren Grenzübergang zwischen der anschaulichen Darstellungsmethode und dem operativen Diskursverfahren wird. Im Erfolgsfalle, der nicht garantiert ist, könne der Grenzübergang nach der logischen Form der Theorie als gleichwertig und nach der Darstellungsmethode als gleichartig beurteilt werden.

Plessners Forschungsauffassung von Wissenschaft weiß sich schließlich mit Kant darin einig, dass die Sprache der Wissenschaften ,keine direkte Abbildfunktion“ hat, sondern durch ein „Modell“ die „Form und Funktion des wirklichen Gegenstandes erklären und anschaulich machen“ müsse. Damit erübrige sich auch der Versuch direkter Abbildung des Subjektiven der Erkenntnis und der Erkenntnistheorie durch ,introspektive Eigenbeobachtung“ und „erlebnismäßige Evidenz“: Erkenntnistheorie müsse fragen, „wie“ - trotz dieses „problematischen Solipsismus“ als Einwand gegen die Behauptung ,intersubjektiver Objektivität“ - doch „überindividuelle Sachlichkeit möglich sei“ (ebd., 30-31).

Ich habe bisher die wichtigsten Stichworte hervorgehoben, unter denen Plessner das Forschungsverfahren moderner Wissenschaft fasst. Für die Entde- 
ckung von Phänomenen und die Erfindung ihrer Darstellungsmethoden wird die ästhetisch reflektierende Urteilskraft funktionalisiert. Für die Gewinnung von Hypothesen und die Erklärung nach Hypothesen wird die teleologisch reflektierende Urteilskraft funktionalisiert. Für die Herstellung des Zusammenhanges beider Forschungsaufgaben, der phänomen- und der hypothesenbezogenen Aufgabe, wird zwischen dem pragmatisch gesehen grammatikalischen und dem logisch gesehen relational operierenden Sprachgebrauch unterschieden. Die Zuordnung zwischen dem anschaulich intuitiven Formalismus und dem diskursiv operativen Formalismus der Forschung erfolge anfangs zufällig und subjektiv, nämlich je nach ihrem Zusammenspiel in Abhängigkeit von der körperlichen oder psychischen Eigenart der beteiligten Forscher. Diese Abhängigkeit werde überwunden, insofern die Anschauungen zur Wahrnehmung schematisiert und die diskursiven Operationen typisiert werden können, bis es zur Vertretbarkeit des persönlichen Zusammenspiels im Sinne der oben genannten Gleichartigkeit und Gleichwertigkeit komme. Um also die moderne Erforschung von Neuem zu ermöglichen, braucht man pragmatisch gesehen ein funktionsteiliges und funktional integrierendes Verfahren, das die reflektierende Urteilskraft regulativ entfaltet, bis die Bedingungen des Ansinnens zugleich die Bedingungen der intersubjektiven und der interindividuellen Einstimmung oder wenigstens der diesbezüglich entschiedenen Stellungnahme geworden sind.

Wenn auf diese Weise moderne Forschung als funktionales Analogon der reflektierenden Urteilskraft verstanden werden kann, hat dies zunächst einen Vorteil gegenüber dem logischen Positivismus als auch im Vergleich mit dem hermeneutischen Problem der Phänomenologie. Statt sich in einen neuen Dualismus zwischen Empirischem und einer transzendentalen Logik hineinzusteigern, stellt Plessner ein pragmatisches Verfahrensmodell heraus, das auf die Einholung der Ermöglichungsbedingungen für die Beurteilung der Zuordnung zwischen dem Diskurs und der Wahrnehmung des Phänomens abhebt. In dieser Ausrichtung des Verfahrens wird zugunsten des wissenschaftlichen Charakters der Forschung als einer intersubjektiven und interindividuellen Unternehmung funktionalisiert, die fehlbar bleibt.

Man kann sich indessen die Funktionalisierung des Verfahrens auch anders vorstellen, nämlich durch eine Proportionierung der Ermöglichungsbedingungen dafür, die Grenzübergänge noch immer beurteilen zu können, nun aber zugunsten der Individualisierung des Vergleichbaren. Das Forschungsverfahren wird dann zugunsten der Kulturwissenschaften und Künste proportioniert, die nicht minder, sondern nur in der Proportionierungsrichtung anders beurteilbar verfahren, wenn sie überhaupt auf moderne Weise vorgehen und nicht nur 
traditionale Feststellungen, gar noch kompensatorischer Art, wiederholen sollen. Plessner verweist ausdrücklich auf das Projekt einer „Kulturwissenschaft“ im Sinne einer vergleichenden Erforschung historisch und gegenwärtig verschiedener Kulturen. Dieser interkulturell vergleichenden Forschung stehe aber die introspektive Selbstbewertung einer einzelnen bestimmten Kultur entgegen, da sie kein indirektes Verfahren der Ermöglichung des Vergleichs durch ein Drittes eröffnet, in welcher Proportionierungsrichtung dann auch immer von der Vergleichsmöglichkeit selber Gebrauch gemacht werde (ebd., 21, 193, 244-245). Das Vergleichbare begegnet auf seiner Grenze dem Unvergleichbaren, d. h. der unteilbaren Einzigartigkeit einer fremden Kultur.

Man könnte für die Frage nach dem in der Moderne auf allen kulturellen Gebieten charakteristischen Forschungsverfahren zusammenfassend sagen, dass Plessner, der mit Max Weber vertraut war, Kants Philosophie systematisch gesehen gleichsam von hinten nach vorne liest, von der dritten Kritik, der Funktionalisierung der reflektierenden Urteilskraft, her. Aus dem daraus gewonnenen Forschungsverfahren folgen die erste und zweite Kritik und die diesen Kritiken entsprechenden Metaphysiken der Natur und der Sitten als historisierbare Resultate, sofern nämlich die früher reflektierende Urteilskraft erfolgreich war und daher inzwischen für die bestimmende Urteilskraft gehalten wird. Das Forschungsverfahren einer bestimmten Proportionierung zeitigt unter historisch endlichen Realisierungsbedingungen endliche Folgen, die sich kulturell sedimentieren. Das Forschungsverfahren der funktionalisierten dritten Kritik kann auch historisch nach kultureller Relevanz nur beschränkt in Gang gesetzt und absolviert werden. So erschienen Kant ein bestimmtes protestantisches Gewissensethos der Innerlichkeit und eine bestimmte Vermögenspsychologie als selbstverständlich. Beide hält Plessner für private und historische Besonderheiten, die sich nicht mehr öffentlich universalisieren lassen und für die es mithin systematisch gesehen funktionaler Äquivalente bedarf. Was

\footnotetext{
[...] in der altmodischen Sprache Kants Vermögen heißt, das bezieht sich dem Wortlaut nach wohl auf Vorstellungen der damaligen Vermögenspsychologie, dem Sinne nach zieht es diese ganz und gar problematischen Anlagen nur heran, um einen Ausdruck zu finden für das Verhältnis zwischen den Feldern, deren Gesetzlichkeit als theoretische, ethische, ästhetische vor Augen liegt, und dem Inbegriff von Gegenständen, auf die sich ihre Begriffe beziehen. (Ebd., 207)
}

Für Plessner geht es nicht mehr um Vermögen, sondern um Leistungen für öffentliche Funktionen in der modernen Gesellschaft und Kultur. 


\subsection{Die Idee des Philosophierens und ihr Ideal der Würde im Widerstreit der Forschungsverfahren untereinander}

Durch die Frage, in welcher Proportion ein Forschungsfeld im Unterschied zu anderen zu einem bestimmbaren und bedingbaren Gebiet funktionalisiert werden sollte, sind wir endlich bei der Frage nach der Spezifik des Philosophischen angekommen.

Was uns zum Vorbild dient und uns die Idee des Verfahrens gibt, wird uns zugleich zum Gegenstand, den wir erkennen sollen. Indem wir die Möglichkeit des Gegenstandes (der exakten Wissenschaft also) begreifen, rechtfertigen wir zugleich die Wahl dieser wissenschaftlichen Methode als Muster für die Philosophie. Natürlich ist das ein Zirkel, wie jedes System uns in einem Zirkel seine Schlüssigkeit beweisen muss. (Plessner 1981e, 20)

Gesetzt, Philosophie könne auch die Form von Forschung annehmen und damit die Idee wissenschaftlicher Philosophie reformuliert werden, indem man das Philosophieren in systematischer Form verteidigt, was unterscheidet sie dann von erfahrungsabhängigen, insbesondere von experimentell beobachtenden Forschungsverfahren?

Derart erfahrungsgewinnenden Forschungsverfahren liege bereits ein Unbedingtes in der Gestalt einer Idee voraus, die regulativ für das Verfahren und in dem Verfahren zum Ideal entfaltet wird, damit dasjenige Gebiet begrenzt werden kann, auf dem nach der Idee Gegenstände zu bestimmen und zu bedingen möglich wird. Das Philosophische entspringe nicht einfach der Beschäftigung mit diesem oder jenem Unbedingten eines bestimmten Forschungsgebietes, das es als sein Unbedingtes voraussetzt, um seine Bedingungen angeben zu können. Das Philosophische entspringe vielmehr dem unvermeidlichen Widerstreit: „Wir verstehen mit Kant unter Antithetik nicht die Lehre irgendeines Gegenteils einer bereits geäußerten und begründeten Lehre, sondern den Widerstreit bestimmter Erkenntnisse untereinander, für die gleiche Rechtsgründe ins Feld geführt werden können, mit der bedeutsamen Einschränkung, dass dieser Widerstreit im Wesen der argumentierenden Vernunft gelegen und daher auch dann unvermeidlich ist, wenn das wissenschaftliche Bewusstsein die Gründe dieses Widerstreits in der logischen und erkenntnistheoretischen Organisation des Urteils und seiner Prinzipien aufgedeckt hat“ (ebd., 152). Philosophie kann demnach „als das Grenzsystem aller Gesetzgebungen in allen Gebieten überhaupt mit Rücksicht auf die Verbindungen zwischen ihnen [aufgefasst werden: HPK], das zwar keine Gesetzgebungen, wohl aber ein gesetzmäßiges Zusammenstimmen unter ihnen bestimmt“ (ebd., 228). 
Wenn es im Philosophieren um ein gesetzmäßiges Zusammenstimmen der miteinander im Widerstreit liegenden Gesetzgebungen bestimmter Forschungsgebiete geht, dann sucht man in ihm „einen unbestimmbaren Begriff, d. i. eine Gesetzmäßigkeit, aber ohne Gesetz, eine Regelmäßigkeit, aber ohne Regel, eine Zweckmäßigkeit ohne Zweck“ (ebd., 222). Anderenfalls würde man die Frage nach dem Zusammenstimmen im Sinne der funktionalen Autonomie eines bestimmten Forschungsgebietes lösen wollen. Es gehe aber nicht um den Widerspruch innerhalb eines bestimmten Forschungsgebietes, sondern um den Widerstreit zwischen ihnen. Die funktionale Autonomie eines bestimmten Forschungsgebietes täte nicht nur den jeweils anderen Forschungsgebieten Unrecht, sondern liefe auch auf den Zirkel der Selbstanwendung einer einzigen Bestimmungsmöglichkeit von Forschung überhaupt hinaus. Plessner weist solche Versuche der Auflösung des Philosophierens durch falsche Verabsolutierungen in Szientismus, Ästhetizismus, Legalismus, Moralismus, Ökonomismus, Historismus, Soziologismus als Versuchungen zur funktionalen Verweltanschaulichung ab, da sie die systematische Herausforderung des Philosophierens aufgeben. Andererseits erscheint ihm auch Kants Versuch, die Lösung in der Heautonomie der Vernunft, d. h. in der Selbsterkenntnis und Selbstgesetzgebung der Vernunft, zu finden, in der problemgeschichtlichen Konstellation, von der ich zu Beginn sprach, wenig überzeugend zu sein.

Plessner rekurriert auch für die Spezifikation des Philosophischen auf die reflektierende Urteilskraft, wobei er die teleologisch reflektierende Urteilskraft als Lösungsorientierung, dem Philosophieren überhaupt eine Idee zu ermitteln, ausscheidet. Sie entpuppe sich entweder als die nur überhaupt reflektierende Urteilskraft im Unterschied zur bestimmenden Urteilskraft oder ihre Teleologie schlage als derart bestimmt und bedingt durch, dass sie für das Problem des Zusammenstimmens der im Widerstreit liegenden Forschungsgebiete nicht in Frage komme (siehe ebd., 213-214). So kritisiert Plessner den „romantischen Stolz" der Marburger Schule, da sie in ihrer teleologischen Orientierung am Organismusbegriff die Autonomie der Philosophie aufgebe (ebd., 249). Übrig bleibe in Kants Systematik allein und erneut die ästhetisch reflektierende Urteilskraft, nach der als funktionalem Analogon die Spezifik der philosophischen Urteilskraft aufzufassen wäre, da es in ihrer Funktionsweise eben um einen unbestimmbaren Begriff, um eine Gesetzmäßigkeit ohne Gesetz, um eine Regelmäßigkeit ohne Regel, um eine Zweckmäßigkeit ohne Zweck gehe. Freilich sei damit noch nicht der „Unterschied von Kunst und Philosophie“ angegeben, da sie in ihrer geschichtlichen Gemeinsamkeit „doch beide Heautonomie der reflektierenden Urteilskraft enthalten“ (ebd., 227). 
Wenn man im ersten Anlauf die philosophische Urteilskraft als Analogon der ästhetisch reflektierenden Urteilskraft versteht, dann ermögliche dies immerhin, dem Philosophieren den Boden zu bereiten. Da die Philosophie nicht wie eine Erfahrungswissenschaft zu einem klar bestimmbaren Gebiet führt, kann die Versprachlichung der dem Philosophieren nötigen Idee nicht durch eine operativ werdende Typisierung des Diskurses erfolgen. Aus demselben Grunde könne auch die Versinnlichung der Idee nicht demonstrativ durch Schematisierung der Anschauung zur Wahrnehmung erfolgen. Vielmehr bedürfe die das Philosophieren ermöglichende Idee eines indirekten Verfahrens ihrer Symbolisierung, von der auch Kant schon sprach, wenn er die Schönheit als Symbol der Sittlichkeit verstand (Kant 1908, § 59). Plessner schließt für die Symbolisierung der Idee des Philosophierens an die sinnvolle Mehrdeutigkeit der Umgangssprache für die Interpretation eines Bildes an.

Plessner schlägt als diesen phänomenal gebbaren und beschreibbaren Ausgangpunkt für die Versinnlichung und die Versprachlichung der Möglichkeit des proportionierbaren Zusammenspiels von Funktionen das „Bild der Würde“ vor: Für eine „Einheit ohne Zweck, mithin formale, aber nicht subjektive, im Gefühl bloß empfundene, sondern zu deutlicher Vorstellung objektiv geeignete, hat die Sprache den Namen der Würde. Würde ist ein Analogon der [körperlich erscheinenden: HPK] Haltung im freien, doch gesetzmäßigen, nur nach einer begrifflosen Regel sich findenden, also zwecklosen Zusammenstimmen aller Vermögen unseres Wesens“ (Plessner 1981e, 247). Im Hinblick auf die Kritik der praktischen Vernunft erläutert Plessner das Bild der Würde als die symbolische Möglichkeit, dass das Wesen des Menschen im Ganzen mit dem höchsten Gute der Disposition nach zusammenstimmen kann: „Zweckmäßig in der Proportion aller Teile im Ganzen (ohne dass das Ganze der Zweck dabei ist), formal in der Zwecklosigkeit der Proportion, objektiv in der Beurteilbarkeit der Proportion in der Erscheinung [als körperliche Haltung: HPK], bietet das Bild der Würde bei einem Menschen zugleich den Ausdruck gediegener Reife im Sinne der Bestimmung der ganzen menschlichen Natur, d. h. in Rücksicht auf das höchste Gut“ (ebd., 248). Dieses Bild, oder besser gesagt, dieser symbolische Rahmen für konkret mögliche Bilder sei aber nicht auf einen bestimmten gegenständlichen Inhalt festgelegt, wodurch sich seine ,ausdrucksvolle Zwecklosigkeit“ in einen bestimmten Zweck mit seinen Mitteln auflösen würde:

Denn wir besitzen in der Erscheinung der Würde, wenn auch kein Schema und keinen Typus, doch etwas, das uns beide in gewissem Sinne, allerdings nur zur inneren Deutlichkeit ersetzt, nämlich ein Symbol, in welchem wir die Art des Verfahrens in der Vorstellung der sinnlichen Erscheinung der Würde auf die unvorstellbare Idee der Arbeit des Menschen im Sinne seiner Bestimmung übertragen. (Ebd., 248) 
Hier geht es nicht um die Bestimmtheit des Menschen, auf die er festgelegt wäre, sondern darum, was er aus ihr infolge seiner eigenen Unbestimmtheit $\mathrm{zu}$ seiner Bestimmung machen kann. Er kann nach dem Prinzip der Harmonie sowohl im Sinnlichen der Erscheinung aller seiner Glieder als auch im Geistigen aller seiner Leistungsfelder ,in der Angemessenheit, dem höchsten Gut und Sinn der Welt zu dienen“ (ebd., 249), leben.

Die Symbolik der Würde ermögliche nicht nur als Idee das Philosophieren, sondern auch als Ideal das Verfahren im Philosophieren $\mathrm{zu}$ orientieren, wenn die Philosophierenden das Symbol der Würde nicht in dieser oder jener inhaltlichen Ausmalung nehmen, die natürlich von Epoche zu Epoche, Kultur zu Kultur, menschlichem Leben zu menschlichem Leben wechselt. „Nur jene Funktion, in welcher der Mensch im Ganzen seiner Existenz ein Bild bietet und eine innere Verfassung zu verstehen gibt [...], nur jene Funktion nennen wir Würde“ (Plessner 1981e, 273). Diese symbolische Funktion könne nun insofern zum Ideal werden, als nach dem exemplarischen Zusammenhang zwischen der Lebensführung des Philosophierenden und der systematischen Form seines Philosophierens gefragt werde. Die systematische Form des Philosophierens ist gegenüber den klar bestimmbaren „Gebietserforschungen“ darin einzigartig, dass sie auf eine „Universalität“ geht, „die in einer bloßen Form liegt, ohne Universalität in der Zusammenfassung von Gegenständen zu sein, die keine endliche Kenntnis je ausfüllen kann“ (ebd., 274). Die reflektierende Urteilsform betreffe den im Hinblick auf Würde strittigen Grenzübergang zwischen bestimmbaren Erforschungsgebieten. Insofern nun die lebensgeschichtliche Frage nach dem „Ganzen aller Vermögen“ der Philosophierenden als ein Analogon der systematischen Form des Philosophierens genommen werden könne, fungiere die symbolische Funktion der Würde als das jeweilige „Philosophieideal“ (ebd., 273).

Dieses Ideal ermögliche uns den spezifisch philosophischen Streit, der sich nun auch der Analogie zum Kunstwerk entzieht. Über Kunstwerke werde gesondert von diesen und von ihren Autorinnen im Diskurs der Kunstkritik gestritten, ohne dass sich die Künstlerin wie der Philosophierende propädeutisch in der öffentlichen Lehre der systematischen Form von Grenzübergängen stellen müsste (siehe ebd., 216-217, 224, 227. 279-280). Der philosophische Streit ermögliche den Vergleich, wie in verschiedenen Kulturen Grenzübergänge im Hinblick auf die symbolische Funktion der Würde gelebt werden, gegebenenfalls bewusst und reflektiert werden. Durch diesen Streit, der so immerhin eine Vergleichsmöglichkeit, wenngleich nicht einen definitiven Maßstab habe, gerät die philosophische Urteilskraft selber in eine Kritik der philosophischen Urteilskraft: 
Ermöglichte die ästhetisch reflektierende Urteilskraft die Idee des Philosophierens, so ermögliche nun die teleologisch reflektierende Urteilskraft das Ideal einer bestimmbaren Philosophie als System. Für die endlich begrenzte Ausführung des Ideals einer bestimmbaren Philosophie, nicht also der unbestimmbaren Idee des Philosophierens überhaupt, teilen die Philosophierenden folgende Annahme: als ob nämlich dieses bestimmte philosophische System die symbolische Funktion der Würde erfüllen könnte (im Konjunktiv). Für die Ausgestaltung dieser systematischen Form des Philosophierens im Unterschied zu anderen Systemen des Philosophierens kommt also doch die teleologisch reflektierende Urteilskraft ins Spiel. „Dass es aber ein Ziel menschlicher Bildung ist, jeder Form menschlicher Würde gerecht $\mathrm{zu}$ werden und sie, jede in ihrer Art, einander gleichzustellen, ist eine Idee, die nur möglich ist auf dem Grunde des Gedankens von der Würde des Menschen überhaupt als einer Form der Beziehung zu dem, was als das höchste Gut zu gelten hat. In diesem grundsätzlichen Liberalismus der formalen Betrachtung liegt ihre Stärke, da kein möglicher Einwand, der in material-gebundener, wirklich-individueller Würde sein Bezugssystem besitzt [...], bei ihr, die alle Einwände überhaupt erst möglich macht, verfangen kann“ (ebd., 275).

Gerade hier, im Kritizismus statt Dogmatismus, wo Plessner Kant am nächsten ist und Kant als den Größten würdigt, ist er ihm auch schon wieder am fernsten, wenn es nämlich um die „Kritik des Philosophierens“ (ebd., 275) selber geht. Kant habe den Widerstreit durch den „Primat der nomothetischen Form“ nicht rational, sondern rationalistisch aus historischen Gründen vorentscheiden wollen: „Den Rahmen aber dieses Kritizismus selbst sprengte Kant nicht. [...] Die Idee der Würde nun entspricht einem offenen, nicht einem geschlossenen System; d. h. gegeben und gefordert ist nur die Idee von einer Proportion aller Vermögen des Geistes unter höchsten Normen und Idealen, eine Architektonik der bloßen Funktion nach [...]. Offen ist das System nur in der Idee; jede wirklich gegebene Systematik ist geschlossen“ (ebd., 278).

Ein geschlossenes System folge einem Ideal von Philosophie, was nötig ist, um im Streit Position zu beziehen, ohne die der Urteilsstreit in der Äußerung privater Vorlieben erlöschen würde. Aber die systematisch verfahrenden Ideale von Philosophie bleiben auf dem Boden des Philosophierens, d. h. im Lichte der Idee von Würde, im Streit. Sie werden geschichtlich durch Kritik von neuem geöffnet. Der geschichtliche Charakter philosophischer Systeme gehöre zu ihrer symbolischen Funktion, nämlich den Widerstreit in Gang zu halten, da die Kritik eines Systems der Philosophie das Philosophieren in systematischer Form erneut herausfordert. Die geschichtliche Differenz zwischen der Idee des Philosophierens und dem Ideal einer systematisch bestimmbaren Philosophie könne 
nicht ihrerseits nochmals in einem System eingefangen oder aus einem solchen begründet werden, sondern werde durch die Kritik aufgedeckt.

Obgleich Plessner Fichte für das Thema der Grenzsetzung und der Grenzübergänge sehr schätzte (Beaufort 2000, VI. Kap.), galt ihm dieser romantische Idealismus gerade als das schlechte Beispiel dafür, durch systematische Deduktionen die geschichtliche Differenz zwischen der Idee des Philosophierens und ihrer Verwirklichung durch ein Ideal einer systematisch bestimmten Philosophie einebnen zu wollen. Fichte dachte

[...] das reine Ich als bloße Spontaneität ohne allen Zusatz, mithin nicht als ein fertig Gegebenes, sondern als Tathandlung, d. i. als ein Übergehen im Vollziehen eines bestimmend-bestimmten Willens. Aber er zog aus dieser Grenzvorstellung der Spontaneität nicht die durch ihr Wesen bedingte Konsequenz, sie so zu begreifen (zu verstehen), wie es ihr als einer Grenze allein angemessen ist. (Plessner 1981e, 98)

Das Tun endlicher Wesen, im Unterschied zur Tathandlung des Absoluten, habe Folgen, die sich diesem Tun gegenüber verselbständigen, weshalb die Aufgabe des Grenzüberganges geschichtlich von Neuem entstehe. Fichtes „Mystik des Tagesbewusstseins“ (ebd., 101) halte nicht ewig an.

Wir haben damit am Ende von Plessners philosophischer Kritik der Urteilskraft eine grammatisch-pragmatische Funktionalisierung der reflektierenden Urteilskraft gewonnen, dieses Mal für die Möglichkeit, die der Spezifik des Philosophierens selber nötig ist. Wenn es das spezifisch philosophische Problem der Grenzziehungen und Grenzübergänge zwischen den verschieden bestimmten Verfahren der Erforschung von Neuem gibt, dann entsteht es durch einen Widerstreit der Verfahren im Hinblick auf die Besetzung einer Idee. Im Falle der Philosophie handele es sich bei dieser Idee um die Symbolik der Würde, die einerseits von einer bestimmten positiven Okkupation frei bleiben muss, andererseits aber die Aufgabe der Bestimmung auf formale Weise freigibt, soll diese Symbolik überhaupt orientieren können. Die pragmatische Funktionalisierung der ästhetisch reflektierenden Urteilskraft ermögliche es, der Idee der Würde einen - für ihre sinnliche Form - bestimmbaren Rahmen möglicher Bilder zu entwerfen. Dessen kategoriale Interpretation werde wiederum durch den heuristischen Konjunktiv der teleologisch reflektierenden Urteilskraft ermöglicht. Auf diese symbolisch indirekte Weise werde nicht nur der Widerstreit zwischen den Forschungsverfahren durch das Philosophieren überhaupt ausgetragen, sondern auch zwischen den verschiedenen philosophischen Systemen selber, nämlich so, als $o b$ sie ihr Ideal und damit die Idee des Philosophierens erfüllen könnten. In der gegenseitigen Kritik der philosophischen Systeme werde die 
Idee des Philosophierens - im Unterschied zu ihren exemplarischen Idealen erneut für die Zukunft geöffnet.

Versteht man klassisch mit Kant unter „transzendental“ die Rekonstruktion der Ermöglichungsbedingungen von Erfahrung im Sinne der physikalischen Wissenschaft, dann hatte schon im Neukantianismus eine Ausweitung der Ermöglichungsfrage auch auf die Erfahrungen der Bio-, Geistes- und Kulturwissenschaften stattgefunden. Plessner versteht diese Ausweitung auf alle möglichen Prozeduren der Erforschung, die durch Technik und Kunst auch andere als wissenschaftliche Unternehmungen betreffen können, noch weiter. Vor allem aber ist seine Art und Weise der Rekonstruktion von Ermöglichungsstrukturen im pragmatischen Sinne auf die Zukunft ausgerichtet. Insofern könnte man seine Habilitationsschrift eine transzendentalpragmatische Untersuchung nennen, die sich später noch weiter zu einer quasi-transzendentalen Rekonstruktion von Lebenserfahrungen entwickeln wird (Krüger 2001). Pragmatisch bedeutet hier aber immer das integrative Zusammenwirken von sinnlichen Anschauungsbedingungen mit Bedingungen der begrifflichen Interpretation gegen die Trennung beider Bedingungsarten. Pragmatisch bedeutet keinesfalls die Unterordnung unter bestimmte Zwecke, für die nur noch die passenden Mittel gesucht werden. Der Widerstreit zwischen den Verfahren der Erforschung entsteht nur im Hinblick auf die Idee der Würde, einer freien Zwecklosigkeit, die sich als Selbstzweck verstehen kann, um bestimmte Zweck-Mittel-Relationen kritisch zu begrenzen.

\subsection{Ausblick auf Plessners spätere Ausgestaltungen der philosophischen Orientierung an der Würde von Personen}

Am Ende dieses Beitrags soll ein Ausblick darauf stehen, wie Plessner seine - in Auseinandersetzung mit Kant gewonnene - philosophische Orientierung an der Würde des Menschen in seinem weiteren Lebenswerk artikuliert. Wenn für Plessner das moderne Philosophieren an die Bewahrung der Würde von Personen gebunden bleibt, was bedeutet die Entfaltung dieser symbolischen Idee dann für sein Ideal in der Sozialphilosophie? Auf diese Frage antwortete er vier Jahre nach seiner Habilitation in seinem Buch Grenzen der Gemeinschaft. Eine Kritik des sozialen Radikalismus (1924, hier Plessner 1981b), in dem er seine Mitmenschen als Personen ansprach. Daran hielt er auch in seinem naturphilosophischen Hauptwerk Die Stufen des Organischen und der Mensch. Einlei- 
tung in die philosophische Anthropologie (1928) ausdrücklich fest (Plessner 1975, 344-345).

Den Menschen als Person zu adressieren, dies hieß historisch zunächst einmal, ihn nicht darauf einzuschränken, nur der Artgenosse oder der Volksgenosse oder der Klassengenosse sein zu dürfen. In dem damaligen Deutschland dominierten Gemeinschaftsideologien, die den einzelnen Menschen darauf reduzierten, diejenige Aufgabe $\mathrm{zu}$ erfüllen, in die er durch seine Familiengemeinschaft hineingeboren und zu der er in einer Berufsgemeinschaft erzogen worden war. Diese notwendigen Gemeinschaftsformen wurden durch ideologische Fehlverallgemeinerung auf die ganze Gesellschaft übertragen, indem man die rassische Volkszugehörigkeit (Nationalsozialismus) oder die soziale Klassenzugehörigkeit (Bolschewismus) als die alles entscheidenden Kriterien der jeweiligen totalitären Bewegung praktizierte (Plessner 1981b, 43). Den Menschen hingegen als Person anzusprechen, dies bedeutet, ihm den Rechtsstatus der Ermöglichung seiner Freiheit zuzuerkennen, die ihn nicht lebenslang auf seine soziale oder vermeintlich biologische Herkunft reduziert. Das Rechtsinstitut der Personalität führt über die Gemeinschaftszugehörigkeiten hinaus in eine zivilisierende „Öffentlichkeit“, d. h. in „das offene System des Verkehrs zwischen unverbundenen Menschen“ (ebd., 95). Sie sind in Gesellschaft - gemessen an ihren Gemeinschaftsbindungen - untereinander Andere und Fremde, die füreinander anders und fremd bleiben können, ohne sich einer bestimmten Gemeinschaft assimilieren zu müssen, die aber auch neue soziokulturelle Relationen des wirtschaftlichen, kulturellen und politischen Austauschs ausbilden können. „Die Gesellschaft lebt allein vom Geist des Spieles“ (ebd., 94). Gesellschaftliche Interaktionen werden in dem Maße (gegenüber purer Gewalt oder dem Sich-aus dem-Wege-Gehen) stabilisierbar, als sie die Funktionswerte spezifischer Verkehrssphären wie der internationalen Politik, Wirtschaft und Zivilisation erfüllen (Plessner ebd., 95). „Alle öffentlichen Relationen beruhen auf dem Prinzip der Gegenseitigkeit“ (Plessner 198bc, 101).

Der angesprochene Gegensatz zwischen Gemeinschafts- und Gesellschaftsformen bedarf einer modernen Lösungsform, die Plessner als gewaltenteiliges Verfahren versteht, das aus den äußeren und inneren Kriegen der Weltgeschichte eine zivilisatorische Lehre zieht. Jedem Menschen kommen als Person die Grundrechte der westlichen Verfassungsgeschichte zu. Der Staat sei „keine Substanz“, sondern ein „Verfahren“, die Ansprüche der Gemeinschaftsformen und der Gesellschaftsformen gegeneinander auszugleichen (Plessner 1981b, 115). Die „Methode dieses Ausgleichs“ bestehe im „Recht“: „Auf der imaginären Schnittgeraden von Gemeinschaftskreis und Gesellschaftskreis liegt das Recht 
als die ewig in Wandlung begriffene Einheit von Gesetzgebung und Rechtsprechung.“(ebd., 116).

Die grundsätzliche Problemstellung in der Sozialphilosophie, ob nämlich die Gemeinschaftsformen über die Gesellschaftsformen oder umgekehrt die Gesellschaftsformen über die Gemeinschaftsformen den Vorrang in der westlichen Moderne beanspruchen können und müssen, ist uns heute auch aus der angloamerikanischen Diskussion des letzten halben Jahrhunderts wohl vertraut. Unter dem Titel des Kommunitarismus wurde ein Gemeinschaftsprimat und unter dem Titel des Liberalismus ein Gesellschaftsprimat vertreten. Wollte man Plessners Position in dieser Diskussion aktualisieren, müsste man seine Methode des rechtsgeschichtlich erkämpften Ausgleichs wohl einen kommunitären Liberalismus des Instituts personaler Grundrechte nennen. Was aber noch immer die Eigenart von Plessners Begründung für den Ausgleich zwischen Gemeinschafts- und Gesellschaftsformen auszeichnet, ist sein Verständnis von der „Unteilbarkeit“ und „Einzigartigkeit“, d. h. kurz von der „Individualität“, der Person. Erst der Ausgleich von Gemeinschaft und Gesellschaft ermögliche die Individualisierung der Person, denn die Individualisierung der Person vor sich selber und vor anderen sei nicht mit der physischen „Vereinzelung“ in ihrem Organismus zu verwechseln (ebd., 61f.). Der Mensch wachse sich nicht irgendwie schon von alleine zum Menschen aus, sei es als Organismus in der Außenwelt oder als Seele in der Innenwelt. Seine Individualität ist nicht als Eigenschaft eines Dinges vorgegeben, er müsse vielmehr in einem lebenslangen Prozess „das Schicksal der Individualisierung auf sich nehmen“ (ebd., 60). Dafür, zu sich selbst erst zu gelangen, bedürfe ein Mensch des Umwegs über Gemeinschafts- und Gesellschaftsformen, aus denen er zu sich kommen kann. Man verwechsele die Individualität von Personen daher auch nicht mit ihrer Eindeutigkeit, denn die Individualisierung einer Person in ihrem lebensgeschichtlichen Prozess werde nur aus ihrer „ontologischen Zweideutigkeit“ her verständlich:

Der doppeldeutige Charakter des Psychischen drängt zur Fixierung hin und zugleich von der Fixierung fort. Wir wollen uns sehen und gesehen werden, wie wir sind, und wir wollen ebenso uns verhüllen und ungekannt bleiben, denn hinter jeder Bestimmtheit unseres Seins schlummern die unsagbaren Möglichkeiten des Andersseins. Aus dieser ontologischen Zweideutigkeit resultieren mit eherner Notwendigkeit die beiden Grundkräfte seelischen Lebens: der Drang nach Offenbarung, die Geltungsbedürftigkeit, und der Drang nach Verhaltung, die Schamhaftigkeit. (Plessner 1981b, 63)

Statt Menschen auf ihre positiven Merkmale zu fixieren und ihnen mithin ihre Freiheit zur Selbstveränderung zu nehmen, bedürfen sie solcher Sozialformen, in denen ihre künftige „Potentialität“, die ihre immer hier und jetzt geleistete 
„Aktualität“ (ebd., 64) übersteigt, gewahrt werden kann. Die personale Lebensform ist fragil, denn sie stellt ihren Angehörigen die Aufgabe, ihre Physis und ihre Psyche, ihren Körper und ihre Seele unter der „Idee einer Harmonie“ zu verschränken. Diese erstrebenswerte Idee ist die der Würde, an der gemessen die Unzulänglichkeit des Hier und Jetzt hervortrete. „Würde betrifft stets das Ganze der Person, den Einklang ihres Inneren und Äußeren“ (ebd., 75). Man muss sie selbst vor Anderen aufführen können und Anderen den Spielraum zu ihrer Aufführung vor einem selbst einräumen können. Sie entsteht nicht, solange man sich im Zirkel der Ehre bewegt, sich allein und selber oder durch Gleichgesonnene „die Lauterkeit seiner Gesinnung“ und „Aufrichtigkeit“ zu bestätigen (ebd., 75). Erst auf dem Umweg des Schauspiels in und mit den Gemeinschafts- und Gesellschaftsrollen für Personen komme man auch als Individuum zu sich. Ohne diesen Weg des Vergleichs mit Vergleichbarem und Unvergleichbarem könne man gar nicht erfahren und verstehen, inwiefern man selber und andere unteilbar und einzigartig sein können (siehe Krüger 1999 u. im vorliegenden Band Zweiter Teil).

Die westlichen Grundwerte von Personen erscheinen rückwirkend als die Resultate einer viel längeren Zivilisationsgeschichte, in der „Zeremoniell und Prestige“ (Plessner 1981b, 79) und „Diplomatie und Takt“ (ebd., 95) erst entwickelt wurden, um aus der Gewaltgeschichte herausgelangen zu können. Seit der römischen Antike bedeutet die persona beides, einerseits das Rechtsinstitut der Freien, andererseits den zivilisierten Status, durch öffentliche Aufführung von Rollenmasken Ansehen zu erlangen. In dieser Zivilisationsgeschichte war der Personenstatus noch auf Oberschichten begrenzt, in ihr konnten aber auch utopische Potentiale für die Universalisierung von Personalität auf alle Menschen ausgebildet werden (ebd., 93-98, 105f.) Um den Eigenwert der Individualität vor sich selber und anderen entwickeln zu können, benötige man als strukturelle Ermöglichung, so Plessner, die Verdoppelung der Person in eine private und öffentliche Person vor anderen Personen in der Mitwelt. Erst die von den Personen geistig geteilte Mitwelt ermögliche die konkrete Erfahrung, „mittels der ersten, zweiten, dritten Person“ in „Einzahl und Mehrzahl“ zueinander und über einander Ich, Du, Er/Sie/Es, Wir, Ihr und Sie sagen zu können (Plessner 1975, 300, 304; vgl. zur Interpretation Krüger 2017d, 188-189, 213-223).

Systematisch gesehen hält Plessner an dem Eigenwert der Individualität von Menschen fest, indem er ihn aus dem Dualismus, entweder in der Außenwelt als organismische Eigenschaft oder in der Innenwelt als seelische Eigenschaft vorkommen zu müssen, befreit. Der Eigenwert der Individualität wird als die lebensgeschichtliche Aufgabe der Individualisierung von Personalität in der Mitwelt begriffen. Dadurch entfällt die einseitige Einsperrung der Individualität 
in die Introspektion der Innenwelt und ihre ebenso einseitige Aussperrung in die naturwissenschaftliche Beobachtung der Außenwelt (vgl. Plessner 1975, 48-50, 67-71). Man steht so nicht mehr vor der Fehlalternative, entweder die Individualisierung durch Atomisierung oder die Vergesellschaftung durch Kollektivierung des Menschen zustande bringen zu müssen. Vielmehr wird die Aufgabe der Individualisierung von Personalität einerseits „horizontal“ in die Relationen zwischen Personen, d.h. in die inter-personalen Relationen, und andererseits „vertikal“ (ebd., 30, 36) in das Verhältnis der Personalität zu Körpern und Leibern, $d . h$. in die intra-personalen Relationen, verlegt.

Da die Person außerhalb ihres Organismus in Relationen zu anderen Personen situiert ist, kann sie zu ihrem Organismus ein doppeltes Verhältnis ausbilden. Sie kann ihn einerseits als Instrument und Medium verwenden, was Plessner kurz das „Körper-Haben“ nennt, in dem die Körper für die Person austauschbar, vertretbar und ersetzbar werden. Andererseits fällt die Person in ihrem lebendigen Vollzug mit ihrem Organismus zusammen, was Plessner kurz das „Leib-Sein“ nennt, in dem ihr Organismus für sie nicht austauschbar, nicht vertretbar und nicht ersetzbar ist, so exemplarisch in ihrem ungespielten Lachen und Weinen (Plessner 1982, 238-241). Demnach sind Personen nicht atomisierte Selbstbewusstseine, sondern Relata von solchen horizontalen und vertikalen Relationen, ohne die sie weder existieren noch verstanden werden können. Wir wachsen in diese historisch geronnenen Unterscheidungen hinein und halten sie daher zunächst für natürlich, obgleich hinter ihnen ein weltgeschichtlicher Prozess an Vermittlungen steht, weshalb sie uns hier und jetzt als eine „vermittelte Unmittelbarkeit“ (Plessner 1975, 321-341; vgl. zur Interpretation Krüger 2017d, 195-208) begegnen.

1931 erfolgte in Plessners Buch Macht und menschliche Natur. Ein Versuch zur Anthropologie der geschichtlichen Weltansicht (Plessner 1981d) die Artikulation des Würdeprinzips in der Gestalt des Prinzips der offenen Frage - oder gleichbedeutend - des Prinzips von der Unergründlichkeit personalen Lebens im Hinblick auf die Geisteswissenschaften (siehe im vorliegenden Band 17. Kapitel). Es handelte sich um die Explikation desjenigen Potentials, das man seit Wilhelm Dilthey die hermeneutische Aufgabe des Verstehens im Unterschied zum Erklären nannte und das Georg Misch dem Prinzip der Unergründlichkeit nach systematisiert hat. Es gehe um das Verstehen von Phänomenen, „die sich selber aussprechen und von sich Zeugnis ablegen“ (Plessner 1981d, 173). Sie bezeugen selbst, wie sie es verstehen, sich zu Verhalten verhalten zu können, indem sie sich ausdrücken und aussprechen, auf den Ausdruck anderer eingehen und sich von diesen ansprechen lassen. Die Geisteswissenschaften können als Wissenschaften nicht auf eine „Garantie der Beantwortbarkeit“ ihrer Fragen 
in dem Sinne verzichten, als ihre Fragen „vernünftig“ und „entscheidbar“ gestellt werden sollten. Sie müssen wohl aber auf die „Garantie der Beantwortung“ ihrer Fragen durch Experiment und Messung verzichten,

[...] da ihre Gegenstände nicht als Erscheinungen, d. h. in Raum- und Zeit-Festlegungen erschöpfbare Größen genommen werden können. Die Unmöglichkeit einer freien Verfügung über ihre Objekte, wie sie das Experiment darstellt, und die Unmeßbarkeit ihrer unräumlichen und unzeitlichen Beschaffenheit findet jedoch in ihrer unmittelbaren Zugänglichkeit oder Verständlichkeit - denn ihre Objekte sprechen sich selber aus und geben sich dem um sie Bekümmerten zu bedeuten - das positive Gegengewicht. (Ebd., 181)

Verglichen mit der naturwissenschaftlichen Beantwortungsgarantie seien die geisteswissenschaftlichen Fragen „offene Fragen“: „eine offene Frage ist, was den Sicherheitsgrad ihrer Entscheidung angeht, der formal geschlossenen Frage des Naturforschers allerdings unterlegen. Dafür zielt sie aber in die Sache selbst, anstatt auf die Regel der eindeutigen Festlegung der Sache“ (ebd., 181).

Die Zeitlichkeit personalen Lebens braucht Versuche, auf die Fraglichkeit dieses Lebens durch Zurechnungen zu antworten.

Schon Ich und Du und Wir sagen können bedeutet in der damit vollzogenen Abgrenzung der Sphären des Mein, Dein, Unser, Aller Zurechnungen, die zwar noch keinen expliziten juristischen Charakter tragen, aber eine rechtliche Ordnung des Lebens in allen seinen Äußerungen nötig machen. (Ebd., 200)

Zurechnungen überhaupt sind in der Thematisierung personalen Lebens erwartbar, doch gegen die moderne Fokussierung des Westens auf das Ich als den Mittelpunkt aller Zurechnungen hebt Plessner zunächst das Du, Wir, Man als gleichberechtigte Möglichkeiten der Modi von Zurechnungen hervor (ebd., 209). Aber selbst diese Ausweitung der Perspektiven auf eine „Verschränkung der Perspektiven im Miteinander und Gegeneinander“ (ebd., 196-197) reicht Plessner angesichts des dem Westen kulturgeschichtlich Fremden nicht: Es sei

[...] dem Menschen zu überlassen - und gewaltlos von ihm zu erfahren, was er ist und als was er sich auslegt, ob er die Essenz seiner Existenz in seine Existenz oder: in einen anderen Menschen, in Tier oder Pflanze, in Stern oder Erde, in einen Gott oder die Elemente setzt. Die vom Abendland errungene Weite des Blicks erfordert die Relativierung der eigenen Position gegen die anderen Positionen. Ihr Mittel und ihre Entdeckung ist der Begriff Mensch, sind im Grunde alle formalen oder formalisierbaren Kategorien wie Leben, Kultur, Welt. Dieser ihrer Relativität hat sich die eigene Position bewusst zu bleiben, wenn sie in der Gleichmöglichkeit des Verstehens und Ausdeutens anderer Positionen die Gefahr der Uniformierung des Fremden nach eigenem Wesensschnitt vermeiden will. (Ebd., 159) 
Nimmt man die geisteswissenschaftliche Frageweise ernst, muss man ihre Beantwortbarkeit von der Antwort des Befragten in seinen geschichtlichen Taten abhängig halten, in denen ihm das „Kriterium für die Richtigkeit“ der Wesensaussage „selbst überantwortet“ wird (ebd., 191). Aber dann besteht die geisteswissenschaftliche Frageweise darin, den Fragenden und Antwortenden einen gemeinsamen Geschichtsprozess in der Zukunft zu ermöglichen. Die Einrichtung der geisteswissenschaftlichen Erkenntnispraxis lässt sich nicht von demjenigen Politischen trennen, das einen gemeinsamen Geschichtsprozess in der Zukunft ermöglicht. Daher verbindet sich für Plessner die geisteswissenschaftliche Frageweise nach den historisch errungenen und historisch veränderbaren Verstehensgründen der Zukunft mit dem politischen Projekt eines pluralen und demokratischen Europas.

In dem Verzicht auf die Vormachtstellung des eigenen Wert- und Kategoriensystems gibt sich der europäische Geist den Horizont auf die ursprüngliche Mannigfaltigkeit der geschichtlich gewordenen Kulturen und ihrer Weltaspekte als einer offenen, unbegrenzten, durch keinen ,Weltgeist‘ planvoll gebundenen Mannigfaltigkeit frei. Diese Universalität des Blicks verlangt die Rücknahme der Verabsolutierung auch ihres Weltaspekts. Spät errungen, wird die geschichtliche Relativierung sich zuletzt ihrer eigenen Relativität bewusst und lernt sie, nach einer Periode historistischer Verzweiflung, nunmehr ruhig als die Bedingung einer echten Objektivität begreifen. (Ebd., 185)

Der Zukunftsgewinn im Verzicht auf den Vorrang des bisher westlichen Selbstverständnisses wird im Sinne einer pluralen Demokratie als des neuen Weltrahmens entworfen, der die politische Referenz von Plessners Philosophischer Anthropologie enthält: „Denn der Begriff des Menschen ist nichts anderes als das ,Mittel', durch welches und in welchem jene wertedemokratische Gleichstellung aller Kulturen in ihrer Rückbeziehung auf einen schöpferischen Lebensgrund vollzogen wird“ (ebd., 186). In Europa hat sich aber bekanntlich nicht der Weg der Wertedemokratie, sondern der Weg der totalen Politisierung in Gestalt der stalinistischen, faschistischen und der nationalsozialistischen Diktaturen durchgesetzt, auf den Plessner mit Carl Schmitt als realer Möglichkeit 1931 verwies (ebd., 141-143, 192-193). Ob Europa das seit 1989-1992 begonnene Projekt einer pluralen Demokratie fortsetzen kann, steht $\mathrm{zu}$ hoffen angesichts des Drucks aus den anderen Zentren einer multipolaren Weltordnung. Die Weltgeschichte steht strukturell erneut vor diesem Spektrum an Möglichkeiten, auf die Herausforderung $\mathrm{zu}$ antworten. Es reicht von der rechts- und sozialstaatlich verfassten Demokratie, in der im Rahmen der konstitutionellen Grundwerte eine Pluralität von Werten gelebt werden kann, wodurch die Frage nach dem Menschen offen bleibt, bis zu neuen Diktaturen, die die Frage nach dem Wesen des Menschen ideologisch schließen. Plessner hält auch in seinem Spätwerk ab den 
1960er Jahren an der Verteidigung der Offenheit und an der Kritik der ideologischen Schließung dieser Frage fest (siehe Plessner 1983e). Die Symbolik der Würde wurde im Zeichen des homo absconditus (Plessner 1983h), d. h. im Prinzip von der Unergründlichkeit des Menschen, bewahrt (siehe Krüger 2013a u. 2015). 